\title{
Fourier Methods for Harmonic Scalar Waves in General Waveguides
}

\author{
Anders Andersson \\ School of Engineering, Jönköping University \\ Börje Nilsson \\ Department of Mathematics, Linnæus University \\ Thomas Biro \\ School of Engineering, Jönköping University
}

June 2, 2015

The final publication is available at Springer via

http://dx.doi.org/10.1007/s10665-015-9808-8

\begin{abstract}
A set of semi-analytical techniques based on Fourier analysis is used to solve wave scattering problems in variously shaped waveguides with varying normal admittance boundary conditions. Key components are newly developed conformal mapping methods, wave splitting, Fourier series expansions in eigen-functions to non-normal operators, the building block method or the cascade technique, Dirichlet-toNeumann operators, and reformulation in terms of stable differential equations for reflection and transmission matrices. For an example the results show good correspondence with a finite element method solution to the same problem in the low and medium frequency domain. The Fourier method complements finite element analysis as a waveguide simulation tool. For inverse engineering involving tuning of straight waveguide parts joining complicated waveguide elements, the Fourier method is an attractive alternative including time aspects. The prime motivation for the Fourier method is its added physical understanding primarily at low frequencies.
\end{abstract}

\section{Introduction}

Scattering of waves in guides of different shapes is a classical problem in applied mathematics. The problem appears in many applications: in acoustics and electrodynamics [19, 18], quantum physics [22], and water waves [1, 2]. Water waves require usually non-linear modelling whereas linear models are often adequate for acoustics and electrodynamics. 
When treating this problem mathematically, the task is to find solutions to the wave equation in some of its forms, and an essential part of this task for linear waves is to solve Helmholtz equation

$$
\left(\nabla^{2}+k^{2}\right) \varphi=0
$$

in different geometries and with various boundary conditions.

For simple geometries, for example straight waveguides with hard or soft walls, the solutions are easily found in terms of Fourier series that is numerically efficient, except for high frequencies, or more precisely, with the exception of situations where the width of the waveguide is much larger than the wavelength. Examples using this technique are found in basic text books on partial differential equations. Straight waveguides with hard or soft walls having abrupt changes in geometry like bifurcations, $90^{\circ}$ bends and open ends can be dealt with using Fourier methods such as Wiener-Hopf and mode matching techniques [26]. By combining these techniques with the Building Block, BB, method [30], also denoted as the generalized scattering matrix [26] and the cascade [19] technique, it is possible to consider sudden area changes [26, 30]. Except for simple waveguide elements like circular bends, see e.g. 9], the treated elements are non-smooth but even for the circular bend, its connection to adjoining waveguides are modelled as nonsmooth [9]. Generalizations to more complicated boundary conditions, like normal surface impedance or admittance, have also been done [11].

The Finite Element method (FE), was originally rarely used for waveguide problems. However, due to evolution of both methods and computers and of a big supply of commercial software, FE is today often the natural choice, even for these problems [34, 16].

Nevertheless, there is a long tradition in using analytic or semi-analytic methods for the solution of two-dimensional static problems, like the harmonic or the bi-harmonic equations, with a complicated geometry using conformal mappings [27]. Such conformal mappings have also been used to improve finite element and finite difference methods to achieve a more efficient and controlled mesh generation [17]. During recent years, numerically efficient conformal mapping algorithms have been developed, first for piecewise linear [14] and then for smooth boundaries [4, 5]. Examples of the application of these or similar numerical conformal mapping methods for waveguides are [3, 6] for time harmonic acoustic and electromagnetic waves and [28] for non-linear time dependent water waves. Here, [3, 6] are based on numerically stable methods for propagation in straight waveguides with varying parameters, see e.g. [15, 31], whereas [28] is based on a stabilizing iterative method.

As mentioned above the BB method can be used for some generalizations in geometry and to further improve the numerical performance, for the semianalytical methods in particular. 
Parallel to this use of conformal mappings for internal propagation in waveguides, quasi-conformal mappings have been used for acoustic and electromagnetic cloaking in exterior domains [32. Coordinate transformations that are not orthogonal but simple to generate have also been used for waveguides. Their common drawback with slow convergence for the solution, due to that homogeneous boundary conditions are transformed into inhomogeneous ones, has recently been removed by adding supplementary modes [25].

Simulation tools for wave propagation have a wide range of applications with varying requirements from the end user. Examples from industrial requirements are:

1. For the analysis of a given design at the end of the design process, it is required that many effects, like complicated geometry and physics, are taken into account with a sufficient accuracy. At this stage, the calculation time has not the highest priority given that it is possible to perform the calculations.

2. In contrast, the accuracy is less important at an initial schematic parameter survey. However, the calculation time might be more critical and the influence of the parameters should be modelled with sufficient care.

3. Quick simulation tools are required in inverse engineering where a design with a specified performance is looked for. The latter can later be checked with a more accurate but slower method. Quick simulation tools are also required in real time applications even at the expense of reduced accuracy.

4. In the search for an optimal design in discussions with engineering colleagues it may be important to include physical interpretations of the simulation. The physical interpretation of the simulations is also important if it is required to generalize the mathematical models by using experimental results.

A successful application of the combination of Fourier methods and the $\mathrm{BB}$ method meeting requirement no 3 is the design of micro wave filters [9]. At that time, commercially available FE softwares were not fast enough to be employed alone but were used to check the found design. Even if FE solvers are much quicker today, the BB method is still very attractive for inverse engineering involving the variation of lengths of straight waveguide parts connecting fixed more complicated waveguide parts. In addition, the BB method improves the physical understanding, requirement 4 in particular if physical modes are used making a natural connection to semi-analytical methods. The need for having a good physical understanding is stressed by 
[28] and also that conformal mappings add physical understanding to the solution with the numerical method.

The wide range of requirements from industry and further requirements from more fundamental research indicate that more than one simulation tool might be motivated. To this end it is interesting to investigate if semianalytical methods based on Fourier analysis can complement FE and FD (finite difference) methods in the simulation of wave models with complicated geometry and general normal impedance or admittance boundary conditions. The prime motivation for these semi-analytical methods, in the sequel called Fourier methods, is the increased physical understanding. Even if it is exceptional, there are cases according to the example above when the Fourier method is the quickest one. Another case with an advantage for the Fourier method is when the domain is naturally described with basis functions having a non-discrete spectrum. One example is the radiation from open pipes, which is conveniently solved with Wiener-Hopf methods, and is naturally linked to other scattering wave guide elements with the BB method. There are even cases when there is not yet a FE solution available. One example is the convective instabilities that may exist for waves in moving fluids [29] or plasmas [10].

The purpose with the current paper is to demonstrate that Fourier methods can solve time harmonic scattering problems in waveguides with complicated geometry and general normal impedance or admittance boundary conditions. This generalization of Fourier methods deals with added complications compared to previous works [25, 31, which assume vanishing normal admittance. Only waveguides with smooth boundaries are considered. Additional well-known tools such as mode matching and Wiener-Hopf methods suitable for handling discontinuities, see for example [19], are necessary to solve general waveguide problems, but are not used in this article. The high frequency regime, for which asymptotic methods exist, is not included.

For the Fourier method, we use a toolbox containing a set of methods:

- The BB method makes it possible to divide a complicated geometry into several tractable parts.

- Different conformal mapping methods are used to further simplify the geometry.

- Reformulation of (11) assuming that the field can be expressed in Fourier series.

- Determination of numerically stable differential equations for reflection and transmission matrices means that these can be determined for each part of the waveguide.

- Dirichlet-to-Neumann operators make it possible to formulate and 
solve numerically stable differential equations for the field in the waveguide.

In an example where the field in the waveguide is scalar, we show how these techniques can be combined, in order to get the complete solution of Helmholtz equation in a two-dimensional waveguide with general geometry and normal admittance boundary conditions. In order to check the accuracy of the method, results are presented not only in the low frequency regime. The results in this example are then compared with the results when using commercial FE software to solve the problem.

The plan of the paper is as follows: In Section 2, the mathematical basis for a Fourier solution is outlined and in Section 3, it is described how the field as well as reflection and transmission matrices are determined in a single "block", using conformal mappings and different reformulations of equation (1). Section 4 shows how these blocks can be combined, using the BB method. Section 5 contains the example problem and a detailed description of the techniques used to solve it. Finally, a discussion and some conclusions are included in Section 6 .

\section{Formulation of the scattering problem}

The problem under investigation is scattering of scalar harmonic waves $\varphi$ in a two-dimensional waveguide $V$ of rather general shape. $\varphi$ solves Helmholtz equation

$$
\left(\nabla^{2}+k^{2}\right) \varphi(\boldsymbol{r})=0, \quad \boldsymbol{r} \in V,
$$

in the interior $V$ of the waveguide and fulfils a homogeneous boundary condition

$$
\frac{\partial \varphi}{\partial n}=\mathrm{i} k \beta \varphi, r \in \partial V
$$

on the boundary $\partial V$. Here, $k \in \mathbb{R}^{+}$is the wave number, $\beta \in \mathbb{C}, \operatorname{Re} \beta \geq 0$ is the (normalised) normal surface admittance and $\widehat{\boldsymbol{n}}$ is the outward pointing normal to $\partial V$. Special cases of the boundary conditions are $\beta=0$ (Neumann or hard) and $\beta=\mathrm{i} \infty$ (Dirichlet or soft). The formulation assumes the time dependence $\exp \left(-\mathrm{i} k c_{0} t\right)$ in the underlying wave equation where $c_{0}$, is the wave speed of the medium in the waveguide. In the formulation (2) 3 ), physical dimensions are omitted. Sources in terms of incident waves will be specified at the end of this section.

The waveguide $V$ consists of three parts: an inner bounded part $V_{\mathrm{i}}$ and two straight semi-infinite parts $V_{\mathrm{L}}$ and $V_{\mathrm{R}}$. An axial co-ordinate $u$ is associated to the waveguide with the positive axis aligned with $V_{R}$ and the negative with $V_{\mathrm{L}}$. The transverse coordinate in the straight waveguides $V_{\mathrm{L}}$ and $V_{\mathrm{R}}$, orthogonal to $u$, is called $v$. Fig. 1 depicts this waveguide schematically. Using the notation $\mathbb{R}_{u_{0}}^{ \pm}=\left\{u \in \mathbb{R}: u \gtrless u_{0}\right\}$, the straight 


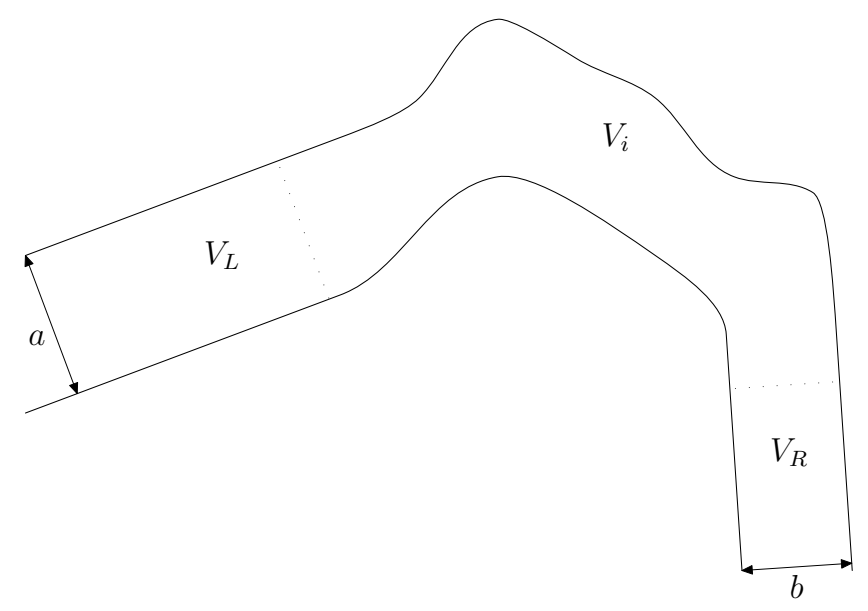

Figure 1: Waveguide $V=V_{L} \cup V_{i} \cup V_{R}$ consisting of straight parts $V_{L}$ and $V_{R}$ open to infinity and a bounded connecting part $V_{i}$.

waveguide parts are $V_{\mathrm{L}}=[0, a] \times \mathbb{R}_{u_{\mathrm{L}}}^{-}$and $V_{\mathrm{R}}=[0, b] \times \mathbb{R}_{u_{\mathrm{R}}}^{+}$. For simplicity it is assumed that the surface admittance $\beta=0$ on $\partial V_{\mathrm{L}} \cap \partial V$ and on $\partial V_{\mathrm{R}} \cap \partial V$, whereas $\beta$ can be non-vanishing and varying on $V_{\mathrm{i}}$.

As a preparation for the definition of the scattering problem, we discuss propagation of waves in an infinite straight waveguide $V_{\gamma}=[0, \gamma] \times \mathbb{R}$, representing $V_{\mathrm{L}}$ if $\gamma=a$ or $V_{\mathrm{R}}$ if $\gamma=b$. If $\varphi$ solves (2) in $V_{\gamma}, \varphi$ can be uniquely splitted into

$$
\varphi(u, v)=\varphi_{-}(u, v)+\varphi_{+}(u, v),
$$

where

$$
\varphi_{ \pm}(u, v)=\sum_{n=0}^{\infty} \varphi_{n}^{ \pm}(u) \psi_{\gamma n}(v), 0 \leq v \leq \gamma .
$$

Here, $\psi_{\gamma n}(v)$ is a basis function, $\varphi_{n}^{ \pm}(u)$ is the corresponding expansion coefficient,

$$
\left\{\begin{array}{l}
\varphi_{n}^{ \pm}(u)=A_{n}^{ \pm} \mathrm{e}^{ \pm \mathrm{i} \alpha_{\gamma n} u} \\
\psi_{\gamma n}(v)=\cos \frac{n \pi v}{\gamma}
\end{array},\right.
$$

$A_{n}^{ \pm}$is a constant modal amplitude and

$$
\alpha_{\gamma n}=\left\{\begin{array}{l}
\sqrt{k^{2}-(n \pi / \gamma)^{2}}, k \geq n \pi / \gamma \\
\mathrm{i} \sqrt{(n \pi / \gamma)^{2}-k^{2}}, k<n \pi / \gamma
\end{array}\right.
$$

is the axial wavenumber.

If $\varphi_{+} \not \equiv 0$, we say that there is a source at $u=-\infty$, and if $\varphi_{-} \not \equiv 0$, there is a source at $u=+\infty$. It makes it natural to denote $\varphi_{-}$or $\varphi_{n}^{-}$as left-going and $\varphi_{+}$or $\varphi_{n}^{+}$as right-going.

In straight waveguide parts like $V_{\mathrm{L}}$ and $V_{\mathrm{R}}$ it is possible to identify the right-going wave $\varphi_{+}(u, v)$ with its coefficient vector $\varphi_{+}(u)=\left(\varphi_{0}^{+}(u), \varphi_{1}^{+}(u), \ldots\right)^{\mathrm{T}}$ 
and $\varphi_{-}(u, v)$ with $\varphi_{-}(u)=\left(\varphi_{0}^{-}(u), \varphi_{1}^{-}(u), \ldots\right)^{\mathrm{T}}$. This coefficient formulation is now used to formulate the scattering problem where the so-called incident waves, the right-going or plus wave in $V_{\mathrm{L}}$ and the left-going or minus wave in $V_{\mathrm{R}}$, are known representing sources in the far left and right portions of the waveguide, respectively. To be determined are the remaining parts of the waves in $V_{\mathrm{L}}$ and $V_{\mathrm{R}}$, denoted the scattered waves. Due to linearity it is possible to relate linearly the known incident waves $\varphi_{\text {inc }}^{+}\left(u_{1}\right)$ and $\varphi_{\text {inc }}^{-}\left(u_{2}\right)$ with the scattered waves $\varphi^{+}\left(u_{2}\right)$ and $\varphi^{-}\left(u_{1}\right)$ using

$$
\left(\begin{array}{l}
\boldsymbol{\varphi}^{+}\left(u_{2}\right) \\
\boldsymbol{\varphi}^{-}\left(u_{1}\right)
\end{array}\right)=\mathcal{S}\left(u_{1}, u_{2}\right)\left(\begin{array}{c}
\boldsymbol{\varphi}_{\text {inc }}^{+}\left(u_{1}\right) \\
\boldsymbol{\varphi}_{\text {inc }}^{-}\left(u_{2}\right)
\end{array}\right), u_{1} \text { in } V_{\mathrm{L}} \text { and } u_{2} \text { in } V_{\mathrm{R}} .
$$

Since the scattered waves are formed from a transmitted and a reflected part, it is convenient to write the scattering matrix $\mathcal{S}\left(u_{1}, u_{2}\right)$ as

$$
\mathcal{S}\left(u_{1}, u_{2}\right)=\left(\begin{array}{ll}
T^{+}\left(u_{2}, u_{1}\right) & R^{-}\left(u_{1}, u_{2}\right) \\
R^{+}\left(u_{2}, u_{1}\right) & T^{-}\left(u_{1}, u_{2}\right)
\end{array}\right)
$$

Here, $R^{-}\left(u_{1}, u_{2}\right)$ and $R^{+}\left(u_{2}, u_{1}\right)$ are reflection matrices, and $T^{+}\left(u_{2}, u_{1}\right)$ and $T^{-}\left(u_{1}, u_{2}\right)$ are transmission matrices.

The scattering matrix for an infinite straight waveguide of width $a$ is

$$
\mathcal{S}\left(u_{1}, u_{2}\right)=\left(\begin{array}{cc}
U_{a}\left(u_{2}-u_{1}\right) & 0 \\
0 & U_{a}\left(u_{1}-u_{2}\right)
\end{array}\right) \text {. }
$$

where

$$
U_{a}(u)=\left(\begin{array}{cccc}
\mathrm{e}^{\mathrm{i} \alpha_{a 0} u} & 0 & 0 & \cdots \\
0 & \mathrm{e}^{\mathrm{i} \alpha_{a 1} u} & 0 & \cdots \\
0 & 0 & \mathrm{e}^{\mathrm{i} \alpha_{a 2} u} & \\
\vdots & \vdots & & \ddots
\end{array}\right) .
$$

All matrices are infinite in size. In numerical calculations, the matrices are truncated. Note, however, that it may be required to include cut-off modes in the calculations.

If the wave field is required in only the straight waveguides $V_{\mathrm{L}}$ and $V_{\mathrm{R}}$, the solution to the above scattering problem is equivalent to the scattering matrix $\mathcal{S}\left(u_{1}, u_{2}\right)$. This scattering matrix approach facilitates understanding and formulation as well as reduces the computational time of scattering problems. The reason is that the scattering matrix for a complicated structure can be synthesized from the scattering matrices for simple structures as will be shown in section 4 .

\section{Solution of the one-block problems}

Wave scattering in a complicated geometry with varying boundary conditions can be treated as a series of simpler problem, using the so called 
Building Block Method, see Section 4. The method determines reflection and transmission matrices for the waveguide, given that these matrices have been determined for each part ("block") of the waveguide.

In this section, we show how reflection and transmission matrices for a single block are established, but also, using a Dirichlet-to-Neumann formulation, how the wave field inside the block could be determined if required. It is assumed that the scattering matrix for each block is determined for anechoic terminations. Otherwise, the interactions due to the termination should have been included in the Building Block Method making it much more complicated. To this end each block is assumed to be an infinitely long waveguide with parallel straight walls and constant boundary conditions outside some bounded transition region. Virtual intermediate straight waveguides, required by the anechoic condition, but with a vanishing length can be inserted [30] demonstrating that the anechoic termination assumption introduces in practice no limitation of the method.

In each such geometry, the boundary value problem

$$
\begin{cases}\left(\nabla^{2}+k^{2}\right) \varphi(x, y)=0 & \text { in the waveguide, } \\ \frac{\partial \varphi}{\partial n}=\mathrm{i} k \beta(t) \varphi & \text { on the boundary }\end{cases}
$$

where $\beta$ varies smoothly with some boundary parameter $t$, should be solved. For the sake of simplicity, we assume that one of the waveguide walls is hard, giving a Neumann boundary condition there.

We use a conformal mapping

$$
F: w=u+\mathrm{i} v \rightarrow z=x+\mathrm{i} y
$$

to transform the geometry into a straight horizontal waveguide $\{u \in \mathbb{R}, 0 \leq v \leq 1\}$ in the $(u, v)$-plane, see Fig. 2. After this transformation, Eq. (12) yields

$$
\left\{\begin{array}{l}
\left(\nabla^{2}+k^{2} \mu(u, v)\right) \Phi(u, v)=0 \\
\left.\frac{\partial \Phi(u, v)}{\partial v}\right|_{v=1}=\mathrm{i} k Y(u) \Phi(u, 1) \\
\left.\frac{\partial \Phi(u, v)}{\partial v}\right|_{v=0}=0
\end{array},\right.
$$

where $\mu(u, v)=\left|F^{\prime}(w)\right|^{2}$ and $Y(u)=\beta(u)\left|F^{\prime}(u+i)\right|$. Note that the boundary condition at the upper boundary, now at $v=1$, is still a normal admittance boundary condition with an admittance that is modified by the scale factor $\left|F^{\prime}(u+i)\right|$. Generally, the new admittance is varying for constant $\beta$ but this variation can be ignored asymptotically at both ends of the waveguide.

Following the techniques, outlined in [6], where an electromagnetic scattering problem is treated, or [31], where similar problems from acoustics are 


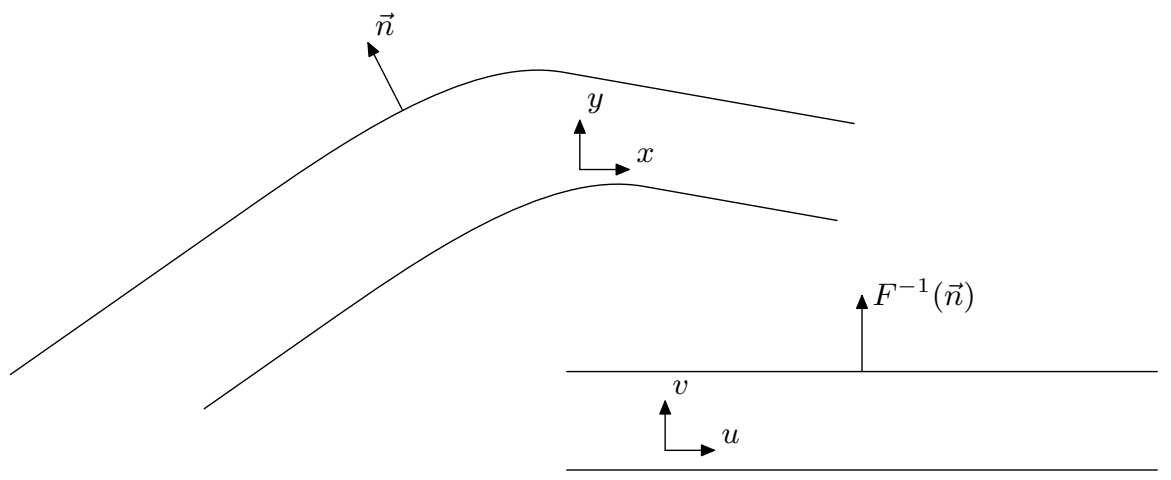

Figure 2: A single block in the $z=x+\mathrm{i} y$ plane and the $w=u+\mathrm{i} v$ plane.

solved, we expand $\Phi(u, v)$ in cosine Fourier series over $v$, assuming that

$$
\Phi(u, v)=\sum_{n} \Phi_{n}(u) \psi_{n}(u, v),
$$

where $\psi_{n}(u, v)=\cos \left(v \lambda_{n}(u)\right)$ for functions $\lambda_{n}(u), \quad n \in \mathbb{N}$.

The functions $\lambda_{n}$ are determined by the boundary condition (13), from which follows that $\lambda_{n}(u), n=0,1, \ldots$, are solutions to the equation

$$
\lambda_{n}(u) \tan \left(\lambda_{n}(u)\right)=-\mathrm{i} k Y(u) .
$$

By differentiating (15), it follows that

$$
\lambda_{n}^{\prime}(u)=\frac{-\mathrm{i} k Y^{\prime}(u)}{Q(u)}
$$

and

$$
\lambda_{n}^{\prime \prime}(u)=-\mathrm{i} k\left(\frac{Y^{\prime \prime}(u) Q(u)-Y^{\prime}(u) Q^{\prime}(u)}{(Q(u))^{2}}\right)
$$

where $(')$ stands for differentiating with respect to $u$ and

$$
Q(u)=\tan (\lambda(u))+\lambda(u)\left(1+\tan ^{2}(\lambda(u))\right) .
$$

From the expansions

$$
\begin{aligned}
& v \sin \left(v \lambda_{n}(u)\right)=\sum_{m} \alpha_{m n}(u) \cos \left(v \lambda_{m}(u)\right), \\
& v^{2} \cos \left(v \lambda_{n}(u)\right)=\sum_{m} \beta_{m n}(u) \cos \left(v \lambda_{m}(u)\right), \\
& \mu(u, v) \cos \left(v \lambda_{n}(u)\right)=\sum_{m} \mu_{m n}(u) \cos \left(v \lambda_{m}(u)\right),
\end{aligned}
$$


follows the infinite-dimensional ordinary differential equation

$$
\boldsymbol{\Phi}^{\prime \prime}(u)-A(u) \boldsymbol{\Phi}^{\prime}(u)-B^{2}(u) \boldsymbol{\Phi}(u)=0,
$$

where the infinite vector $\boldsymbol{\Phi}=\left(\Phi_{1} \Phi_{2} \Phi_{3} \ldots\right)^{T}$ and the infinite matrices $A$ and $B^{2}$ have elements

$$
A_{m n}(u)=2 \alpha_{m n}(u) \lambda_{n}^{\prime}(u)
$$

and

$$
B_{m n}^{2}(u)=\alpha_{m n}(u) \lambda_{n}^{\prime \prime}(u)+\beta_{m n}(u)\left(\lambda_{n}^{\prime}(u)\right)^{2}+\delta_{m n}\left(\lambda_{n}(u)\right)^{2}-k^{2} \mu_{m n}(u) .
$$

Note that $\left(\psi_{m}\right)$, with $\psi_{m}(v)=\cos v \lambda_{m}(u)$, is in general not an orthogonal system for constant $u$ since the eigenvalue problem (13) is a regular Sturm-Liouville problem only if $Y$ is purely imaginary. However, $\left(\psi_{m}\right)$ and $\left(\overline{\psi_{m}}\right)$, with a bar - denoting the complex conjugate, form a biorthogonal system meaning that the bilinear form

$$
\left\langle\psi_{m}, \psi_{n}\right\rangle=\left(\psi_{m}, \overline{\psi_{n}}\right)=\int_{0}^{1} \psi_{m}(v) \psi_{n}(v) \mathrm{d} v
$$

vanishes if $n \neq m$. Here, for complex functions $f$ and $g,(f, g)$ denotes the complex scalar product and the bilinear form $\langle f, g\rangle$ is formally the same as the real scalar product. This means that the expansion coefficients like $\alpha_{m n}(u), \beta_{m n}(u)$ and $\mu_{m n}(u)$ in (18, 20), are found from

$$
a_{m}=\frac{\left\langle f, \psi_{m}\right\rangle}{\left\langle\psi_{m}, \psi_{m}\right\rangle}
$$

for the expansion

$$
f=\sum_{m} a_{m} \psi_{m}
$$

Furthermore, the system $\left(\psi_{m}\right)$ is proved to be a basis in $\mathrm{L}^{2}(0,1)$ [33]. An alternative proof can be given based on the existence of a sequence biorthogonal to $\left(\psi_{m}\right)$ using a theorem by Bari [8], see [13] for a modern presentation of this theorem. It is interesting to note that the biorthogonal property (24) in a complex Hilbert space with general complex $Y$ can be seen as an analytic continuation of the orthogonality property in a real Hilbert space with purely imaginary $Y$.

\subsection{Conformal mapping techniques}

There are two indispensable requirements on the conformal mapping. The first requirement is anechoic terminations at the ends of each block. This is accomplished by treating the block as an infinite waveguide which is straight and has constant cross-sections outside some bounded region. We must therefore numerically construct a conformal mapping from a straight infinite 
waveguide to an infinite waveguide in which the walls at both ends are (at least) asymptotically straight and parallel.

The second requirement relates to smoothness. To avoid singularities in the matrices $A$ and $B^{2}$ in the differential equation (21), it follows from (13) that the mapping must have a bounded first derivative on the boundary, and additionally, from (16), (17), (22) and (23), bounded second and third derivatives if the boundary has non-zero admittance.

In [4] and [5], conformal mapping techniques, suitable for this situation, are developed. Both methods are built on the Schwarz-Christoffel mapping, which guarantees that the resulting waveguide walls are asymptotically straight and parallel towards infinity, and they both result in regions with smooth boundary curves, meaning that no singularities are introduced by the mapping. In [4], a suitable polygon, surrounding the region under consideration, is determined, and the conformal mapping is constructed using the Schwarz-Christoffel mapping for that polygon. In [5], the factors in a Schwarz-Christoffel mapping are replaced by so called approximate curve factors that round the corners in a way that gives a smooth boundary curve.

\subsection{Re-transformation to stable equations}

The differential equation (21) cannot be solved directly by numerical methods. However, there exist reformulations of (21) that are numerically stable. In this section, we describe two such reformulations, built on two different partitions of the wave field $\boldsymbol{\Phi}$.

Recall that the block is assumed to be an infinitely long waveguide which is straight and has parallel hard boundaries outside some central transition region. Let $\Omega_{L}$ and $\Omega_{R}$ be the straight regions to the left and right respectively. In $\Omega_{L}$ and $\Omega_{R}$, the matrix $A$ is zero, while $B$ is constant. Assume that $B=B_{-}$in $\Omega_{L}$ and $B=B_{+}$in $\Omega_{R}$. In $\Omega_{L}$ and $\Omega_{R}, B^{2}$ is a real constant diagonal matrix, and to be consistent with standard theory for straight waveguides, the square roots of $B^{2}$ are chosen such that $B_{-}$and $B_{+}$have either positive real or negative imaginary diagonal elements.

\subsubsection{Determination of the Reflection and Transmission matrices - The RT method}

Inspired by the partition $\varphi=\varphi_{-}+\varphi_{+}$in a straight waveguide where the two terms can be seen as representing waves marching from left to right and right to left respectively, we make the following definition: Let for all $u \in \mathbb{R}$, the wave field

$$
\boldsymbol{\Phi}(u)=\left(\Phi_{1}(u) \Phi_{2}(u) \ldots\right)^{T}=\boldsymbol{\Phi}^{+}(u)+\boldsymbol{\Phi}^{-}(u),
$$

where $\boldsymbol{\Phi}^{+}(u)$ and $\boldsymbol{\Phi}^{-}(u)$ represent waves marching to the right and left respectively. 
Let furthermore $C$ and $D$ be matrices, depending on $u$, such that

$$
\frac{\partial \boldsymbol{\Phi}}{\partial u}(u)=-C(u) \boldsymbol{\Phi}^{+}(u)+D(u) \boldsymbol{\Phi}^{-}(u),
$$

for all $u \in \mathbb{R}$. $C$ and $D$ can be defined in many different ways, but they must be differentiable with respect to $u$, and since (21) must hold in $\Omega_{L}$ and $\Omega_{R}$ where $A(u)=0$, it follows that $C=D=B_{-}$in $\Omega_{L}$ and $C=D=B_{+}$ in $\Omega_{R}$. We have used the definition

$$
C(u)=D(u)=B_{-}+f(u)\left(B_{+}-B_{-}\right),
$$

where $f$ is a smooth function that is 0 in $\Omega_{L}$ and 1 in $\Omega_{R}$.

Define, like in (9), reflection and transmission matrices $R^{+}, R^{-}, T^{+}, T^{-}$, such that for $u_{1}<u_{2}$,

$$
\left(\begin{array}{l}
\boldsymbol{\Phi}^{+}\left(u_{2}\right) \\
\boldsymbol{\Phi}^{-}\left(u_{1}\right)
\end{array}\right)=\left(\begin{array}{ll}
T^{+}\left(u_{2}, u_{1}\right) & R^{-}\left(u_{1}, u_{2}\right) \\
R^{+}\left(u_{2}, u_{1}\right) & T^{-}\left(u_{1}, u_{2}\right)
\end{array}\right)\left(\begin{array}{l}
\boldsymbol{\Phi}^{+}\left(u_{1}\right) \\
\boldsymbol{\Phi}^{-}\left(u_{2}\right)
\end{array}\right) .
$$

This means that $T^{-}$and $R^{-}$transmits respectively reflects the left-going waves $\boldsymbol{\Phi}^{-}$, while $T^{+}$and $R^{+}$transmits respectively reflects the right-going waves $\boldsymbol{\Phi}^{+}$.

From (21), (27) and (28), it is possible to derive, for details see for example [31, the equation

$$
\frac{\partial}{\partial u}\left(\begin{array}{l}
\boldsymbol{\Phi}^{+} \\
\boldsymbol{\Phi}^{-}
\end{array}\right)=\left(\begin{array}{cc}
J & K \\
L & M
\end{array}\right)\left(\begin{array}{l}
\boldsymbol{\Phi}^{+} \\
\boldsymbol{\Phi}^{-}
\end{array}\right)
$$

where

$$
\begin{aligned}
& J=(C+D)^{-1}\left(-C^{\prime}-B^{2}+(A-D) C\right), \\
& K=(C+D)^{-1}\left(D^{\prime}-B^{2}-(A-D) D\right), \\
& L=(C+D)^{-1}\left(C^{\prime}+B^{2}-(A+C) C\right), \\
& M=(C+D)^{-1}\left(-D^{\prime}+B^{2}+(A+C) D\right) .
\end{aligned}
$$

For the determination of $T^{+}$and $R^{+}$, we consider (30) assuming that there are no sources in $\Omega_{R}$. Let $u_{2} \in \Omega_{R}$ be constant and let $u=u_{1}$ vary. This means that $\boldsymbol{\Phi}^{-}\left(u_{2}\right)=0$, and (30) simplifies to

$$
\left\{\begin{array}{l}
T^{+}\left(u_{2}, u\right) \boldsymbol{\Phi}^{+}(u)=\boldsymbol{\Phi}^{+}\left(u_{2}\right), \\
R^{+}\left(u_{2}, u\right) \boldsymbol{\Phi}^{+}(u)=\boldsymbol{\Phi}^{-}(u) .
\end{array}\right.
$$

By differentiating (33) with respect to $u$, we get

$$
\left\{\begin{array}{l}
\frac{\partial T^{+}}{\partial u}\left(u_{2}, u\right) \boldsymbol{\Phi}^{+}(u)+T^{+}\left(u_{2}, u\right) \frac{\partial \boldsymbol{\Phi}^{+}}{\partial u}(u)=0 \\
\frac{\partial R^{+}}{\partial u}\left(u_{2}, u\right) \boldsymbol{\Phi}^{+}(u)+R^{+}\left(u_{2}, u\right) \frac{\partial \boldsymbol{\Phi}^{+}}{\partial u}(u)=\frac{\partial \boldsymbol{\Phi}^{-}}{\partial u}(u),
\end{array}\right.
$$


and using (31) and (33) once more, the Ricatti equations

$$
\begin{aligned}
\frac{\partial R^{+}}{\partial u}\left(u_{2}, u\right)=-R^{+}\left(u_{2}, u\right)(J(u)+ & \left.K(u) R^{+}\left(u_{2}, u\right)\right) \\
& +L(u)+M(u) R^{+}\left(u_{2}, u\right) \\
\frac{\partial T^{+}}{\partial u}\left(u_{2}, u\right)=-T^{+}\left(u_{2}, u\right)(J(u)+ & \left.K(u) R^{+}\left(u_{2}, u\right)\right)
\end{aligned}
$$

follow. For $R^{-}$and $T^{-}$, we proceed similarly assuming no sources in $\Omega_{L}$, and deduce the equations

$$
\begin{aligned}
\frac{\partial R^{-}}{\partial u}\left(u, u_{1}\right)=-R^{-}\left(u, u_{1}\right)(M(u)+ & \left.L(u) R^{-}\left(u, u_{1}\right)\right) \\
& +K(u)+J(u) R^{-}\left(u, u_{1}\right), \\
\frac{\partial T^{-}}{\partial u}\left(u, u_{1}\right)=-T^{-}\left(u, u_{1}\right)(M(u)+ & \left.L(u) R^{-}\left(u, u_{1}\right)\right) .
\end{aligned}
$$

Using truncated matrices in place of $J, K, L$ and $M$, these equations can be solved numerically with an ordinary differential equation solver. (35) and (36) ) are solved from right to left using $R^{+}\left(u_{2}, u_{2}\right)=0$ and $T^{+}\left(u_{2}, u_{2}\right)=I$ as initial values, while (37) and (38) are solved from left to right, using $R^{-}\left(u_{1}, u_{1}\right)=0$ and $T^{-}\left(u_{1}, u_{1}\right)=I$ as initial values.

It is readily shown, see e.g. [31] for more details, that the described procedure for calculating the transmission and reflection matrices is numerically stable.

\subsubsection{Determination of the fields - The DtN method}

To determine the field inside a single block, we reformulate (21) using Dirichlet-to-Neumann matrices, see also [24] and [15]. For this purpose, we make a different partition of $\boldsymbol{\Phi}$. Let

$$
\boldsymbol{\Phi}=\boldsymbol{\Phi}_{R}+\boldsymbol{\Phi}_{L},
$$

where $\boldsymbol{\Phi}_{R}$ are waves with no sources to the right (in $+\infty$ ) and $\boldsymbol{\Phi}_{L}$ are waves with no sources to the left (in $-\infty)$. Define Dirichlet to Neumann (DtN) matrices $\Lambda_{R}$ and $\Lambda_{L}$ such that

$$
\begin{aligned}
& \boldsymbol{\Phi}_{R}^{\prime}(u)=-\Lambda_{R}(u) \boldsymbol{\Phi}_{R}(u), \\
& \boldsymbol{\Phi}_{L}^{\prime}(u)=\Lambda_{L}(u) \boldsymbol{\Phi}_{L}(u) .
\end{aligned}
$$

$\boldsymbol{\Phi}_{R}$ and $\boldsymbol{\Phi}_{L}$ are both satisfying (21), and by differentiating (40) and (41), the matrix equations

$$
\begin{aligned}
& \Lambda_{R}^{\prime}(u)=\left(A(u)+\Lambda_{R}(u)\right) \Lambda_{R}(u)-B^{2}(u) \\
& \Lambda_{L}^{\prime}(u)=\left(A(u)-\Lambda_{L}(u)\right) \Lambda_{L}(u)+B^{2}(u)
\end{aligned}
$$


follow. Since $A=0$ in $\Omega_{L}$ and $\Omega_{R}$,

$$
\begin{array}{lll}
\boldsymbol{\Phi}_{R}^{\prime}(u)+B_{-} \boldsymbol{\Phi}_{R}(u)=0, & \boldsymbol{\Phi}_{L}^{\prime}(u)-B_{-} \boldsymbol{\Phi}_{L}(u)=0, & u \in \Omega_{L}, \\
\boldsymbol{\Phi}_{R}^{\prime}(u)-B_{+} \boldsymbol{\Phi}_{R}(u)=0, & \boldsymbol{\Phi}_{L}^{\prime}(u)+B_{-} \boldsymbol{\Phi}_{L}(u)=0, & u \in \Omega_{R},
\end{array}
$$

which means that if truncated matrices are used in place of $A$ and $B^{2}$, (42) and (43) can be solved numerically from right and left respectively, using the initial values $\Lambda_{R}\left(u_{2}\right)=B_{+}$and $\Lambda_{L}\left(u_{1}\right)=B_{-}$, where $u_{1} \in \Omega_{L}$ and $u_{2} \in \Omega_{R}$. Finally, (40) and (41) are solved numerically from left and right respectively.

We have noted for our example that the Riccati equations (42,43) in the DtN method may be stiff for certain values of $u$ but the stiffness is numerically tractable. This kind of stiffness, not observed for (35 38), is likely connected to a singularity of $\Lambda_{R}(u)$ or $\Lambda_{L}(u)$ for a complex value near the real $u$-axis, see for example [15]. A singularity on the real $u$ axis for $\Lambda_{R}$ can be avoided by alternating between formulations for $\Lambda_{R}, \Lambda_{R}^{-1}$ and $\left(\Lambda_{R}-d I\right)^{-1}$, where $d$ is real and $I$ is the identity matrix, see [23]. Another alternative to handle the singularities is to use recent numerical methods by which Riccati matrix equations are integrated across singularities, even when no knowledge about existence or location of these is at hand, see for example [21].

Numerical problems can also appear at a discrete set of frequencies $k$ for methods based on field calculations, like the DtN method, when trapped modes exist. In this case, the scalar field can for mathematical reasons be determined only modulo an unknown factor times the trapped mode. However, the scattering matrices can be determined with the methods proposed in [31] also in the presence of trapped modes.

\section{Combination of the Blocks - the Building Block Method}

The Building Block method (BB), see [30], allows the determination of reflection and transmission matrices for a combination of several sections ("blocks") of the waveguide, for which these matrices are known.

Assume that two subsequent blocks $\Omega_{1}$ and $\Omega_{3}$, are connected by a region $\Omega_{2}$ which is straight with length $\ell$ and width $a$, see figure 3 . Furthermore, assume that reflection and transmission matrices for $\Omega_{1}$ and $\Omega_{3}$ are known.

To harmonize with the notation in the RT method, we choose here to build on the expansion coefficients $\boldsymbol{\Phi}(u)=\boldsymbol{\Phi}^{+}(u)+\boldsymbol{\Phi}^{-}(u)$ as varying with the position along the waveguide rather than on modal amplitudes that are constant in a straight waveguide. The latter procedure is the most common one in the BB method. The reflection and transmission matrices are defined as follows. Assume that a block begins and ends at position $u_{j}$ and $u_{j+1}$ respectively and that there are no sources at the $u_{j+1}$ side of the block. If 


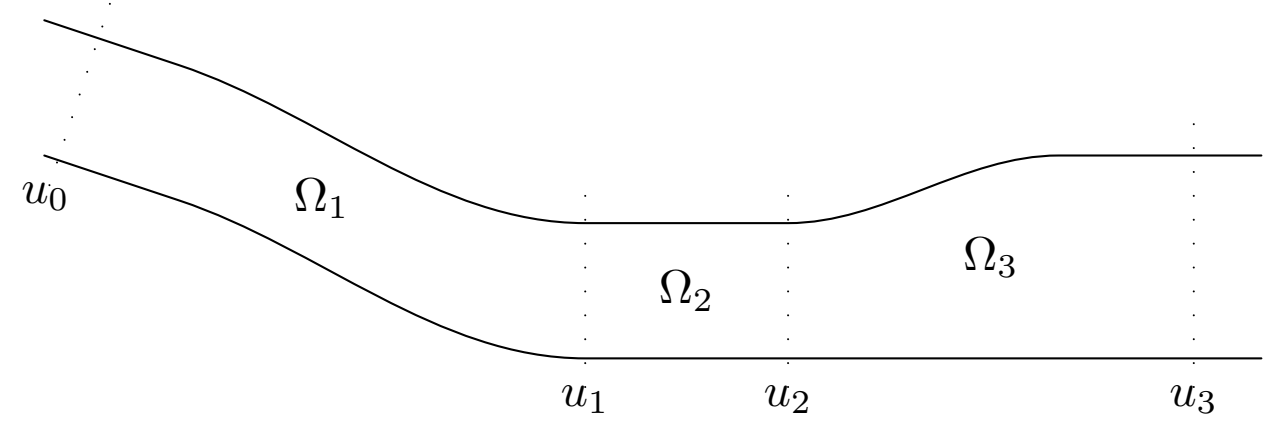

Figure 3: Waveguide divided in blocks. $\Omega_{2}$ is straight and with constant cross-section.

$T^{+}\left(=T^{+}\left(u_{j+1}, u_{j}\right)\right)$ is the transmission matrix for waves $\boldsymbol{\Phi}^{+}(u)$ entering the block at $u_{j}$, then $\boldsymbol{\Phi}^{+}\left(u_{j+1}\right)=T^{+} \boldsymbol{\Phi}^{+}\left(u_{j}\right)$ are the waves leaving the block at position $u_{j+1}$. Let $u$ be a parameter, measuring the distance along the central curve of the waveguide, and let $u=u_{0}$ at the beginning (left end) of $\Omega_{1}, u=u_{1}=0$ at the border between $\Omega_{1}$ and $\Omega_{2}, u=u_{2}=\ell$ at the border between $\Omega_{2}$ and $\Omega_{3}$, assuming that the length of $\Omega_{2}$ is $\ell$, and finally $u=u_{3}$ at the end of $\Omega_{3}$. Following the definitions given in Eq. (30), we use the notation

$$
\begin{array}{ll}
R_{1}^{+}=R^{+}\left(u_{1}, u_{0}\right), & T_{1}^{+}=T^{+}\left(u_{1}, u_{0}\right), \\
R_{1}^{-}=R^{-}\left(u_{0}, u_{1}\right), & T_{1}^{-}=T^{-}\left(u_{0}, u_{1}\right), \\
R_{3}^{+}=R^{+}\left(u_{3}, u_{2}\right), & T_{3}^{+}=T^{+}\left(u_{3}, u_{2}\right), \\
R_{\text {tot }}^{+}=R^{+}\left(u_{3}, u_{0}\right), & T_{\text {tot }}^{+}=T^{+}\left(u_{3}, u_{0}\right) .
\end{array}
$$

Assume that a right-marching field $\boldsymbol{\Phi}^{\text {in }}=\boldsymbol{\Phi}^{+}\left(u_{0}\right)$ is entering $\Omega_{1}$ from the left, and that there are no sources to the right of $\Omega_{3}$. Define matrices $C^{ \pm}$such that at the border between $\Omega_{1}$ and $\Omega_{2}, \boldsymbol{\Phi}^{+}(0)=C^{+} \boldsymbol{\Phi}^{\text {in }}$ and $\boldsymbol{\Phi}^{-}(0)=C^{-} \boldsymbol{\Phi}^{\mathrm{in}}$. Standard theory for straight waveguides gives that in $\Omega_{2}$ at position $u$, the field is

$$
\boldsymbol{\Phi}(u)=\left(U_{a}(u) C^{+}+U_{a}^{-1}(u) C^{-}\right) \boldsymbol{\Phi}^{\mathrm{in}},
$$

where $U_{a}$ is defined as in Eq. (11) in Section 2, Consequently, at the border between $\Omega_{2}$ and $\Omega_{3}$, there are right-marching and left-marching waves $U_{a}(\ell) C^{+} \boldsymbol{\Phi}^{\text {in }}$ and $U_{a}^{-1}(\ell) C^{-} \boldsymbol{\Phi}^{\text {in }}$ respectively.

Since there are no sources to the right of $\Omega_{3}, U_{a}^{-1}(\ell) C^{-}=R_{3}^{+} U_{a}(\ell) C^{+}$, so $C^{-}=U_{a}(\ell) R_{3}^{+} U_{a}(\ell) C^{+}$. But $C^{+}=T_{1}^{+}+R_{1}^{-} C^{-}$, and hence

$$
\begin{aligned}
& C^{+}=\left(I-R_{1}^{-} U_{a}(\ell) R_{3}^{+} U_{a}(\ell)\right)^{-1} T_{1}^{+}, \\
& C^{-}=U_{a}(\ell) R_{3}^{+} U_{a}(\ell) C^{+} .
\end{aligned}
$$

In summary the reflection and transmission matrices for the combined 


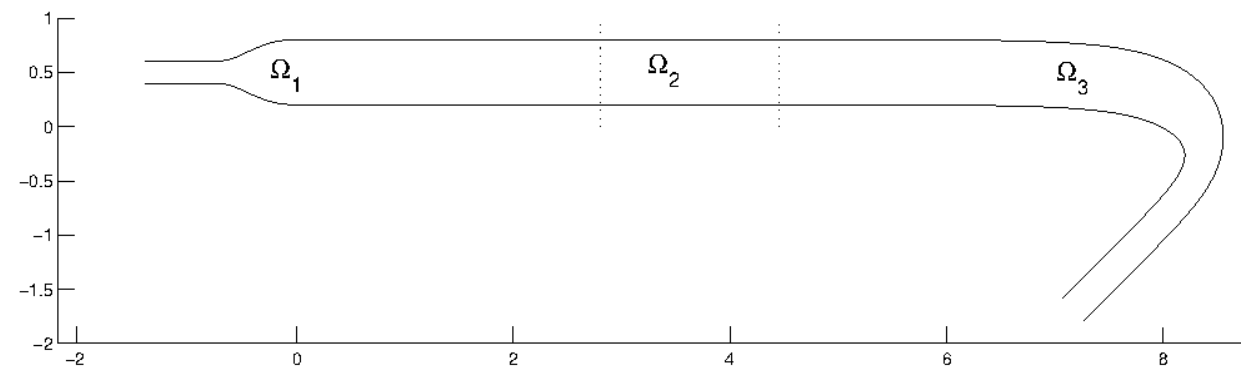

Figure 4: The waveguide in the example.

waveguide $\Omega_{1}, \Omega_{2}$ and $\Omega_{3}$ is

$$
\begin{aligned}
& T_{\text {tot }}^{+}=T_{3}^{+} U_{a}(\ell) C^{+}, \\
& R_{\text {tot }}^{+}=R_{1}^{+}+T_{1}^{-} C^{-} .
\end{aligned}
$$

The method has been known since the end of the 1940:s [20] and is denoted the Building Block Method [30] in acoustic theory and cascade technique [19] in electromagnetic theory.

\section{A numerical example}

To illustrate the techniques, we solve the scattering problem in the waveguide shown in Fig. 4. The results, i.e., the total field in the waveguide given a known field entering from the left, as well as reflection and transmission operators for the waveguide, are calculated and compared with a finite element method solution.

\subsection{Boundary conditions}

The boundaries are hard, i.e., they have zero normal admittance except for two intervals on the upper boundary. In both $\Omega_{1}$ and $\Omega_{3}$, there is admittance at the upper boundary in the intervals $F_{j}([-2,2]+\mathrm{i})$ for $j \in\{1,2\}$. The admittance varies smoothly along the border, and has a maximal level $\beta=$ $0.5+0.5 \mathrm{i}$ in the intervals $F_{j}([-1,1]+\mathrm{i})$, where the functions $F_{j}$ are the conformal mappings defined in Section 5.2 .

\subsection{Conformal mappings}

The region is divided into three disjoint parts. $\Omega_{1}$ contains the change in cross-section to the left, the middle section $\Omega_{2}$ is straight with constant cross-section, and $\Omega_{3}$ contains the bending to the right.

For $\Omega_{1}$, shown in Fig. 5(a), a conformal mapping is constructed using the approximate curve factor technique developed in [5]. The conformal 

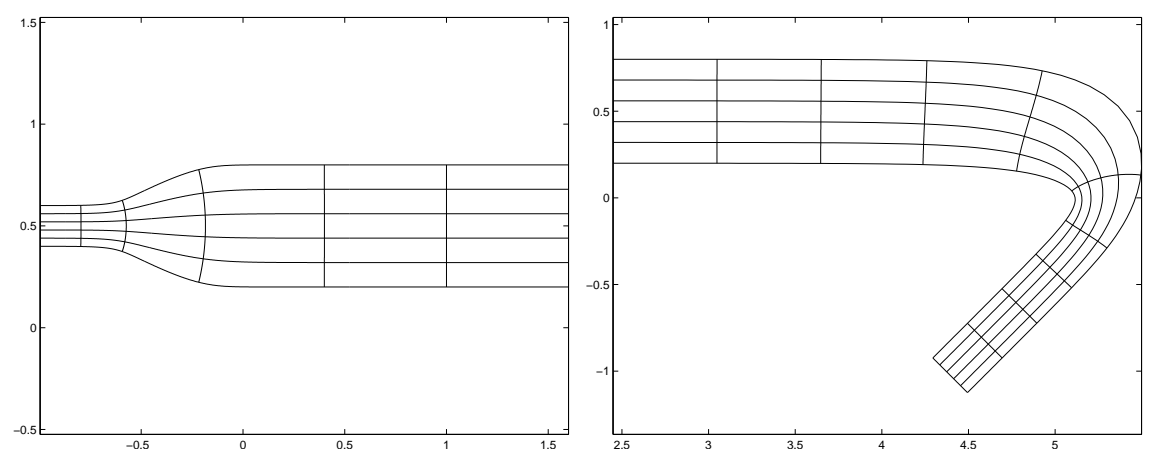

Figure 5: The two building blocks. The grid lines are images under the conformal mappings of $u=-5,-4, \ldots, 4,5$ and $v=0,0.2, \ldots, 1$.

mapping is $F_{1}=f_{1} \circ g_{1}$, where

$$
f_{1}(w)=A \int_{w_{0}}^{w} \prod_{j=1}^{4}\left(\sqrt{\left(\omega+b_{k} \mathrm{i}-w_{k}\right)^{2}-c_{k}^{2}}-b_{k} \mathrm{i}\right)^{\alpha_{k}-1} \omega^{-1} d \omega+z_{0},
$$

and

$$
g_{1}(w)=\exp (\pi w)
$$

In (49), $A=0.6 / \pi$ to get the width 0.6 to the right, $\alpha_{1}=\alpha_{4}=0.85$ and $\alpha_{2}=\alpha_{3}=1.15$ to get inner angles of sizes $1.15 \pi$ and $0.85 \pi, b_{1}=b_{4}=$ $c_{1}=c_{4}=1$ and $b_{2}=b_{3}=c_{2}=c_{3}=0.05$ to get the corners appropriately rounded, while $w_{1}=-1, w_{2}=-a, w_{3}=a$ and $w_{4}=1$, where $a=0.008740$ has been numerically determined to get the width 0.2 to the left. Finally, $w_{0}$ is set to 2 and $z_{0}$ to $1+0.2 i$ to position the waveguide in the complex plane.

For $\Omega_{3}$, shown in Fig. [5(b), the outer polygon method [4] is used to construct the conformal mapping. The mapping function is here $F_{2}=f_{2} \circ g_{2}$, where

$$
f_{2}(w)=A \int_{g_{2}\left(w_{0}\right)}^{w} \frac{(\omega-1)^{\alpha-1}}{(\omega+1)^{\alpha-1}(\omega-a)} d \omega+z_{0}
$$

and

$$
g_{2}(w)=w^{\left(\varphi_{2}-\varphi_{1}\right) / \pi} \mathrm{e}^{\mathrm{i} \varphi_{1}}+a,
$$

with $A=0.1501 \exp (3 \pi \mathrm{i} / 4), \alpha=7 / 4, \varphi_{1}=3 \pi / 10, \varphi_{2}=7 \pi / 10, a=$ $-0.4632, w_{0}=-7$ and $z_{0}=4.4485+0.2 \mathrm{i}$.

As has been mentioned in Section 3, the blocks are in theory assumed to be of infinite length. When determining the reflection and transmisson matrices for one block, it must have sufficiently long straight parts in both ends. The requirement is here that the derivative of the conformal mapping must be practically constant. In this example, it is sufficient to use $-5 \leq$ $u \leq 5$ in $\Omega_{1}$ and $-7 \leq u \leq 7$ in $\Omega_{3}$. Hence, in the calculations, $\Omega_{1}=$ $F_{1}([-5,5]+i[0,1])$ and $\Omega_{3}=F_{2}([-7,7]+i[0,1])$. 


\subsection{Determination of the field and of reflection and trans- mission matrices}

The fields inside $\Omega_{1}$ and $\Omega_{3}$ have been determined using the techniques described in Section 3.2.2. Simultaneously, reflection and transmission operators for $\Omega_{1}$ and $\Omega_{3}$ have been determined using the techniques in Section 3.2.1. All calculations have been made using truncated matrices in the differential equations (35)-(38) and (40,43) and a standard numerical ODE solver (ode45).

We have assumed a source at infinity to the left resulting in a rightmarching wave $\boldsymbol{\Phi}_{\text {in }}=\left(\begin{array}{lllll}1 & 0 & 0 & 0 & \ldots\end{array}\right)^{t}$ entering the waveguide from the left. No sources to the right are assumed.

The matrices $A(u)$ and $B^{2}(u)$ in (21), as well as the matrices $J, K, L$ and $M$ in (32) have been determined for $u=-5,-4.99, \ldots, 5$ in $\Omega_{1}$, and for $u=-7,-6.99, \ldots, 7$ in $\Omega_{3}$. Linear interpolation was then used in the ODE solvers to determine $J, K, L$ and $M$ in eqs. (35) 38) and $A$ and $B^{2}$ in eqs. (42) and (43) for $u$ values not in this set.

Finally, the field in $\Omega_{2}$ as well as reflection and transmission matrices for the whole waveguide where calculated using the Building Block method described in Section 4 .

\subsection{Results}

The methods have been applied for $0<k a \leq 4$ using $10 \times 10$ matrices, where $a=0.2$ is the width of the waveguide at the left end. The real part of the three lowest modes inside the waveguide in Figure 4 is shown in Figure 6 for the incident wave $\boldsymbol{\Phi}^{\mathrm{in}}=\left(\begin{array}{lllllllll}1 & 0 & 0 & 0 & 0 & 0 & 0 & 0 & 0\end{array}\right)^{t}$. For the lowest frequency, only the lowest mode is present except near the waveguide modifications, where also the second mode is visible. For higher frequencies, the situation is more complicated with more propagating modes.

Of special interest is the power lost at the boundary where the admittance has a non-vanishing real part. The power of mode $\Phi_{n}$ with complex amplitude $A_{n}$ in a waveguide with width $a$ is proportional to

$$
\begin{cases}\frac{a\left(1+\delta_{n 0}\right)}{2 k}\left|A_{n}\right|^{2} \sqrt{k^{2}-\left(\frac{n \pi}{a}\right)^{2}}, & k \geq \frac{n \pi}{a}, \\ 0, & \text { otherwise. }\end{cases}
$$

The power ratio $\mathcal{P}$ for the waveguide is defined as the sum of transmitted and reflected power over incident power. $\mathcal{P}$ is less or equal to 1 for a passive structure and less than 1 for passive structure with attenuation. In Figure 7 , $\mathcal{P}$ is shown as a function of $k$. For the chosen frequency independent normal admittance there are losses present for all frequencies, at least $25 \%$ in the low frequency end stabilizing at about $90 \%$ for $k$ larger than 8 . This corresponds to a total attenuation of about $1 \mathrm{~dB}$ and $10 \mathrm{~dB}$, respectively. 

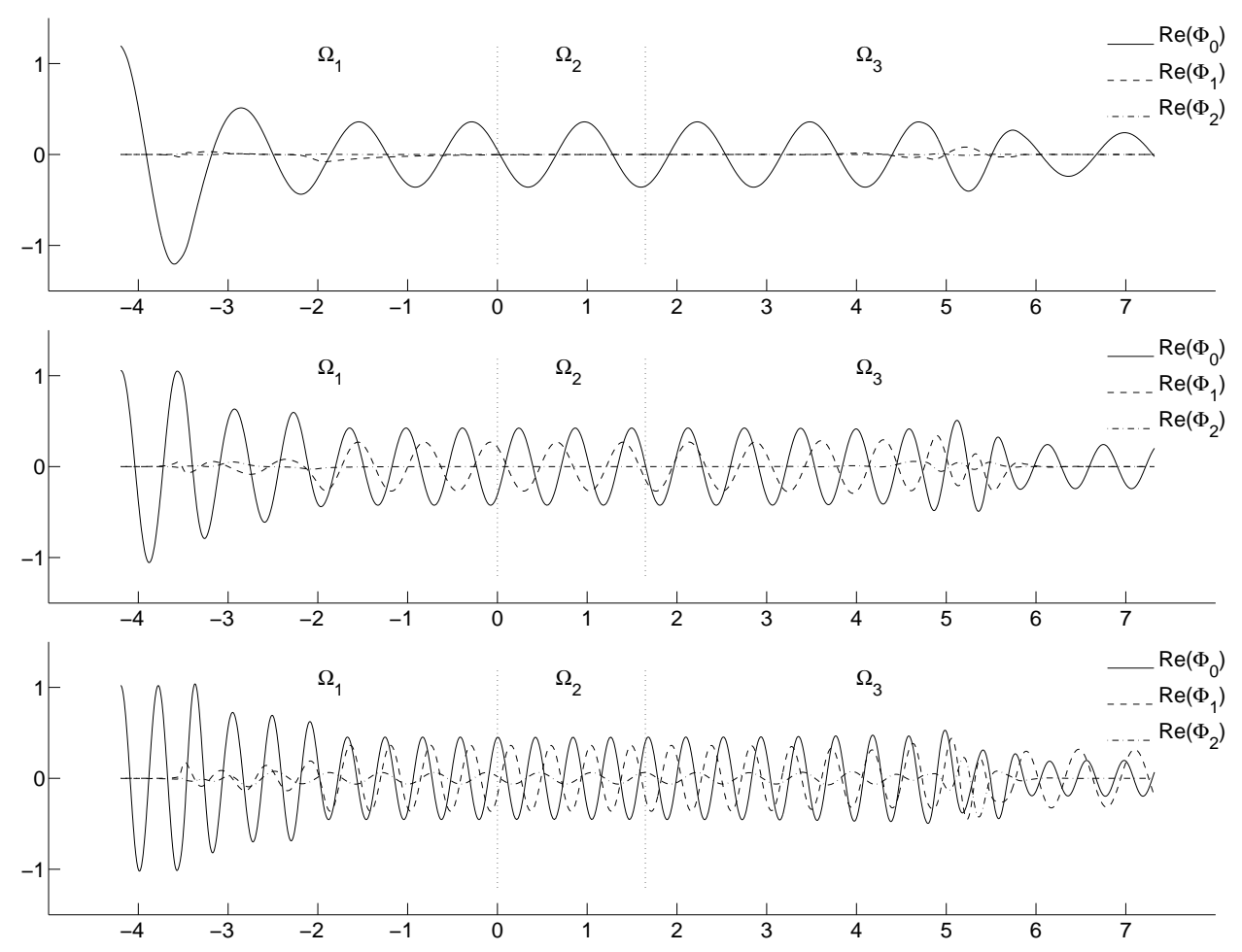

Figure 6: $\operatorname{Re} \Phi_{0}, \operatorname{Re} \Phi_{1}$ and $\operatorname{Re} \Phi_{2}$, are plotted for $k=5, k=10$ and $k=15$ for the incident wave $\boldsymbol{\Phi}^{\mathrm{in}}=\left(\begin{array}{lllllllll}1 & 0 & 0 & 0 & 0 & 0 & 0 & 0 & 0\end{array}\right)^{t}$. The measure on the horizontal axis is the distance along the central curve in the waveguide, coinciding with the $x$-axis in Figure 4 for the interval $(-4,7)$. Dotted vertical lines indicate borders between $\Omega_{1}, \Omega_{2}$ and $\Omega_{3}$. For $k=5(k a=1)$, only $\Phi_{0}$ is propagating (without attenuation) in the three straight parts of the waveguide, while for $k=10(k a=2), \Phi_{1}$ propagates in $\Omega_{2}$ but not in the straight part of $\Omega_{3}$. For $k=15(k a=3)$ finally, all three modes are propagating in $\Omega_{2}$ and $\Phi_{0}$ and $\Phi_{1}$ propagate in the straight part of $\Omega_{3}$.

\subsubsection{Comparison between the RT and DtN methods}

In this section, the RT and DtN methods are compared. The DtN method described in Section 3.2 .2 is used in $\Omega_{1}$ and $\Omega_{3}$, whereas the reflection and transmission matrices from the RT method are used in the BB method, described in Section 3.2.1, for $\Omega_{2}$ and is an alternative to the DtN method for the right end of $\Omega_{3}$. Both methods should give identical results at the border between $\Omega_{1}$ and $\Omega_{2}$ and at the right end of $\Omega_{3}$. For the first case, $\boldsymbol{\Phi}$ using the DtN method is compared with $\left(C^{+}+C^{-}\right) \boldsymbol{\Phi}^{\text {in }}$. Likewise, for the second case $\boldsymbol{\Phi}$ with the DtN method is compared with $T_{\text {tot }}^{+} \boldsymbol{\Phi}^{\text {in }}$. Here, $C^{ \pm}$is determined using (47) and $T_{\text {tot }}^{+}$using (48).

The smooth curves visualized in Figure [6 indicates that for the three 


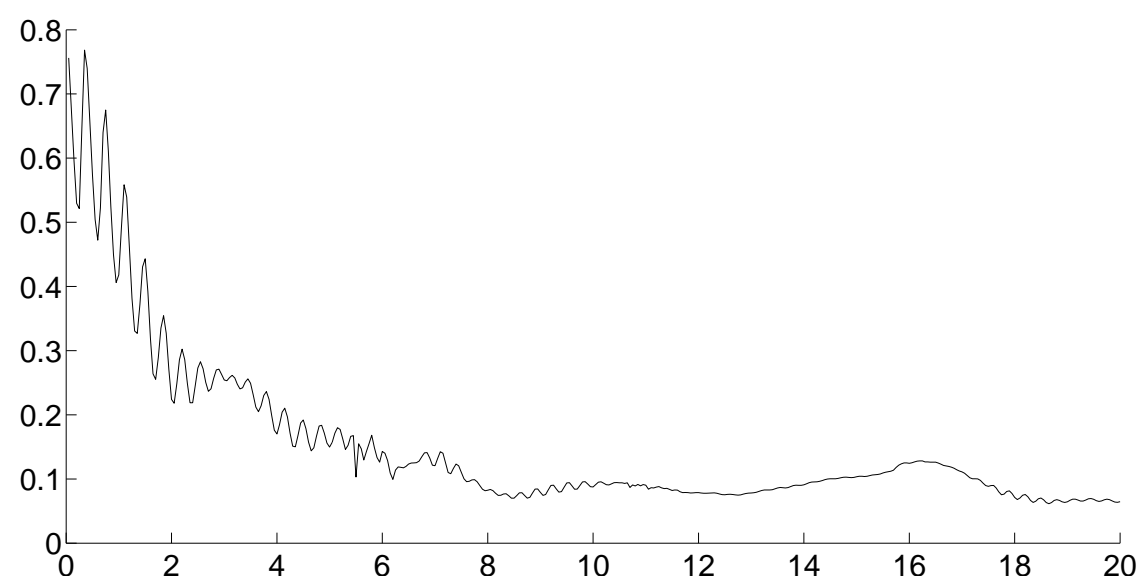

Figure 7: Power ratio $\mathcal{P}$ as function of $k$

$k$ values presented there, the correspondence is good. Similar results are obtained all over the investigated interval $0<k \leq 20$, no matter if only one or if several propagating modes are present at the two points in the waveguide. One example of a more detailed comparison is given in Table 1 , where results for $k=15$ are compared.

\begin{tabular}{|l|rrl|}
\hline & \multicolumn{1}{|c}{$\boldsymbol{\Phi}_{\Omega_{1}}(\mathrm{end})$} & \multicolumn{1}{c|}{$\boldsymbol{\Phi}_{\Omega_{2}}(0)$} & $\mid$ difference \\
\hline$\Phi_{1}$ & $0.4521-0.0448 \mathrm{i}$ & $0.4521-0.0449 \mathrm{i}$ & $3.744 \cdot 10^{-5}$ \\
$\Phi_{2}$ & $-0.1873-0.2693 \mathrm{i}$ & $-0.1873-0.2693 \mathrm{i}$ & $7.971 \cdot 10^{-6}$ \\
$\Phi_{3}$ & $0.0190+0.0203 \mathrm{i}$ & $0.0190+0.0203 \mathrm{i}$ & $7.730 \cdot 10^{-6}$ \\
\hline & \multicolumn{1}{|c}{$\boldsymbol{\Phi}_{\Omega_{3}}(\mathrm{end})$} & $T_{\text {tot }}^{+} \boldsymbol{\Phi}^{\text {in }}$ \\
\hline$\Phi_{1}$ & $0.0671-0.1847 \mathrm{i}$ & $0.0671-0.1848 \mathrm{i}$ & $2.792 \cdot 10^{-5}$ \\
$\Phi_{2}$ & $-0.1926+0.2528 \mathrm{i}$ & $-0.1926+0.2528 \mathrm{i}$ & $2.812 \cdot 10^{-5}$ \\
$\Phi_{3}$ & $\approx 0$ & $\approx 0$ & \\
\hline \multicolumn{4}{|}{} \\
\hline
\end{tabular}

Table 1: Comparison of the RT and DtN method. Above: $\Phi_{1}, \Phi_{2}$ and $\Phi_{3}$ at the border between $\Omega_{1}$ and $\Omega_{2}$ calculated with the two different methods. Below: $\Phi_{1}$ and $\Phi_{2}$ at the end of $\Omega_{3}$ calculated with the two different methods. All calculations are made for $k=15(k a=3)$ and with $10 \times 10$ matrices.

\subsubsection{Comparison with a FE solution}

As a comparison, we have applied a commercial FE solver (COMSOL Multiphysics) to our model problem. In Figures 8 11, FE solutions for $0<k \leq 20$ are compared with the corresponding Fourier method solutions. From the FE solution, it is easy to extract the base mode $\Phi_{0}$ by calculating the average field over a cross-section of the waveguide. In Figure 8 , the FE calculated $\Phi_{0}$ 


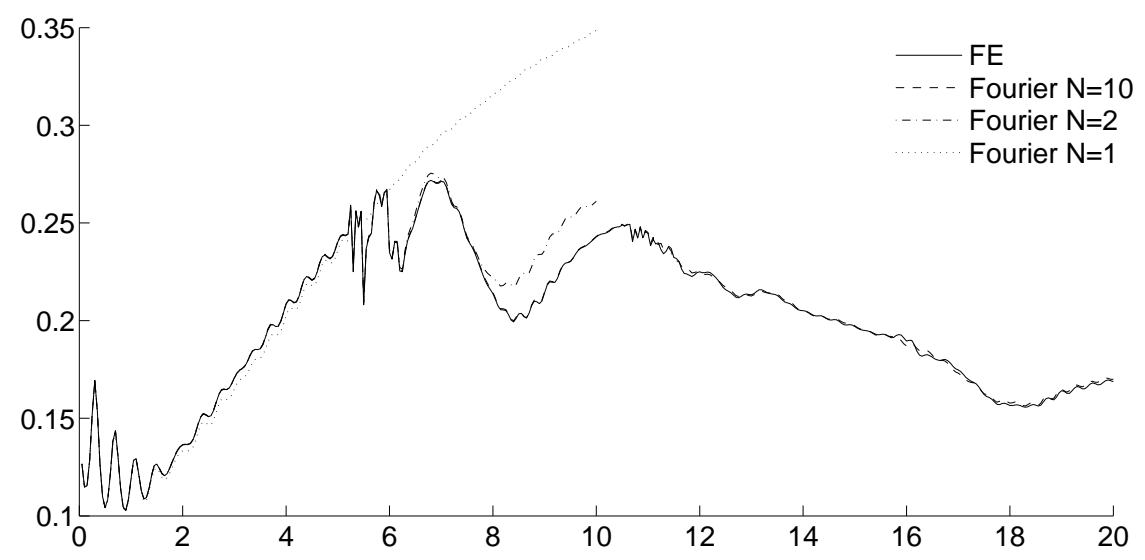

Figure 8: $\left|\Phi_{0}\right|$ at the end of $\Omega_{3}$ calculated for $k=0.05,0.1, \ldots, 20$ with the Finite Element Method and with the Fourier methods described in the article with $N=1,2$ and 10, i.e., using $1 \times 1,2 \times 2$ and $10 \times 10$ matrices in the calculations.

on the boundary at the end of the waveguide is compared with $\Phi_{0}$ calculated with Fourier methods using three different matrix sizes.

For low frequencies, Figure 8 indicates that accurate results can be achieved with small matrices. The situation is examined further in Figure 9 where a relative difference between the FE solution and Fourier solutions using $1 \times 1,2 \times 2$ and $3 \times 3$ matrices are shown.

The width of the waveguide is 0.6 in the central part, which means that there, the second mode $\Phi_{1}$ is propagating (without attenuation) for $k>\pi / 0.6 \approx 5.24$. Clearly, calculations with $1 \times 1$ matrices can not give accurate results for $k>\pi / 0.6$. However, it is interesting to note that even when using only one matrix element in the calculations, fairly good results are achieved for frequencies with not more than one propagating mode. An important feature of the Fourier method is its ability for low frequencies to partition the wave field into a low number of left and right going waves adding valuable physical understanding.

For $k>\pi /(0.2 \sqrt{2}) \approx 11.1$, i.e. when the second mode is propagating without attenuation in the end of the waveguide, the differences are significantly greater, see Figures 10 and 11. Note however that for $k$ just above the frequency 11.1, there are significant interference effects between the two propagating modes. For these frequencies, the problem is ill-posed since the interference is very sensitive to small changes in not only $k$ but also in geometry and boundary conditions. Due to that there are inevitable small differences in the two models, the discrepancies shown in Figures 10 and 11 are therefore not surprising. The main reason for this ill-posedness is that the axial wavenumber of the second mode is small increasing from zero when it starts propagating. Note also that the differences tend to vanish for 
higher frequencies. In $\Omega_{3}$, there are only forward-marching waves present. Additionally, due to the Neumann boundary condition at the end of $\Omega_{3}$, the first mode is constant over the cross-section, while the second mode is at maximum at the boundary. Therefore, the interference is large at the chosen point. At points inside the waveguide, the differences between the two methods are much smaller.

For higher frequencies in the investigated interval, an indication of the required matrix size is given in Figure 12, where the problem is solved for $k=15$ using matrix sizes from $2 \times 2$ upto $50 \times 50$. The results are stable to three significant figures for matrix sizes $7 \times 7$ and upwards.

\section{Discussion and conclusion}

The example problem in Section 5 is of course not a "general" waveguide. There are numerous possible boundary variations not commented so far. For example, the methods in this article seem to require smooth changes in both geometry and boundary conditions to get converging Fourier series. However, the Building Block method and well-established mode-matching techniques can overcome most such problems. Furthermore, as is illustrated in [31] where an L-bend is investigated, good results can be achieved even when the conformal mapping functions contain singularities on the boundary. It is however evident that the differential equations get stiffer and that larger truncated matrices are required.

Another simplification in this article is the assumption of a Neumann boundary condition on one of the boundaries. An iterated use of the Building Block method and lengthways partitions of the waveguide can handle twodimensional waveguides with non-hard walls on both sides.

As a reference and comparison, the problem in the example has been solved using commercial software for the finite element method. As was seen in Figures 8 11, the correspondence between a FE solution and the Fourier methods solution is good, with a small tendency to growing discrepancy with growing $k$. This is not surprising; to maintain a certain accuracy when $k$ increases, both methods require enhanced numerical work. In FE, a finer mesh is needed, for details see for example [16], while the Fourier methods require larger matrices.

When working with $10 \times 10$ matrices, the Fourier method described in this article is very slow compared to the FE method. To produce the results presenterd in Figure 8, i.e., to solve the problem for 400 different $k$ values, takes between one and two hours with the FE method on an ordinary PC, while the copmputation time for the Fourier method was several days on the same machine. In the FE method, the mesh was set fine enough to give accurate results in the higher parts of the investigated frequency interval.

It should of course be mentioned that no professional computer pro- 
grammer has been involved in writing the code for the Fourier routines. We expect that there are still many possibilities to optimize these programs for increased speed.

Note also that for the greater part of the investigated frequency interval $0<k \leq 20$ in our model example, accurate results are achieved with matrix sizes smaller than $10 \times 10$. Indeed, truncating the matrices so that they contain only one element gives fairly good results in intervals where only one mode is propagating. And when using $1 \times 1$ matrices, even unprofessional computer programs perform well.

The most time-consuming part of the calculations is the determination of the matrices $A$ and $B^{2}$ in (21) for a large set of $u$-values. For every value of $u, \lambda_{n}(u)$ for $n=0, \ldots, N-1$ should be found by solving equation (15) numerically. The values of $\alpha$ and $\beta$ in eqs. (18) and (19) can be determined analytically, but for every $u, N^{2}$ numerical integrations are needed to determine the values of $\mu_{m n}(u)$ in Eq. (20). For values of $u$, corresponding to hard boundaries, $\lambda_{n}=n \pi$ and the $\mu$ coefficients can be calculated using Fast Fourier Transforms, but for values of $u$ corresponding to boundaries with admittance, a comparatively slow numerical integration must be used for each matrix element.

A possible drawback for the FE method is that it needs more computer memory in order to determine the wave field for higher frequencies. If we consider an object that consists of a couple of complicated blocks that are in arbitrary order repeatedly connected with straight waveguides, the Fourier method could be an attractive alternative when considering computation time.

Another drawback for some FE commercial packages, not present in the Fourier method, is the treatment of low frequency electromagnetic waves. This is a (solvable) [7, 12] deficiency.

For time harmonic wave scattering problems, the Fourier methods described in this article are applicable for the low and medium frequency domain. It is beyond the scope of this paper to develop more precise bounds for this domain. The method adds physical interpretation, in particular at low frequencies, like partition of the wave field into left and right going physical modes and facilitates a power loss analysis also into terms of modes.

In summary, we conclude, based primarily on requirements from industry, that more than one type of time harmonic waveguide simulation tool is required. It is demonstrated that Fourier methods based on Fourier Analysis provides one such tool. Its accuracy is checked against FE simulations for a general two-dimensional waveguide with normal admittance boundary conditions at low and medium frequencies. For the current investigation with non-zero normal boundary admittance, the Fourier method, with its present implementation, is considerably slower than the FE method that is more memory demanding. However, for inverse engineering involving tuning of straight waveguides, the Fourier method is an attractive alternative 
including time aspects. The prime motivation for the Fourier method is its added physical understanding primarily at low frequencies.

\section{References}

[1] M. R. Abbott. A theory of the propagation of bores in channels and rivers. Proc. Camb. Phil. Soc., 52:344-362, 1956.

[2] S. Aimen, T. Michelle, and W. Theodore. Propagation of solitary waves through significantly curved shallow water channels. J. Fluid Mech., 362:157-176, 1998.

[3] Anders Andersson. Using a zipper algorithm to find a conformal map for a channel with smooth boundary. In AIP Conference Proceedings: 2nd Conference on Mathematical Modeling of Wave Phenomena, volume 834, pages 3-12. AIP, 2006.

[4] Anders Andersson. Schwarz-Christoffel mappings for nonpolygonal regions. SIAM J. Sci. Comput., 31(1):94-111, 2008.

[5] Anders Andersson. Modified Schwarz-Christoffel mappings using approximate curve factors. J. Comp. Appl. Math., 233(4):1117-1127, 2009 .

[6] Anders Andersson and Börje Nilsson. Electro-magnetic scattering in variously shaped waveguides with an impedance condition. In $A I P$ Conference Proceedings: 3rd Conference on Mathematical Modeling of Wave Phenomena, volume 1106, pages 36-45. AIP, 2009.

[7] F. P. Andriulli, K. Cools, F. Olyslager, and E. Michielssen. The dottrick tdefie: A dc stable integral equation for analyzing transient scattering from pec bodies. In IEEE Int. AP-S Symp., volume 4, 2008.

[8] N. Bari. Sur les systèmes complets de functions orthogonales. Mat. Sbornik, 14(56):51-108, 1944.

[9] T. Biro and B. Nilsson. Multiple scattering of microwaves from septum discontinuities in a circular bend. Wave Motion, 42:109-125, 2005.

[10] R. J. Briggs. Electron-stream interaction with plasmas. Number 29 in Res. Monogr. M.I.T. Press, Cambridge, Mass., 1964.

[11] A. Büyükaksoy and G. Cinar. Solution of a matrix wienerhopf equation connected with the plane wave diffaction by an impedance loaded parallel plate waveguide. Mathematical Methods in the applied Sciences, 28:1633-1645, 2005. 
[12] W. C. Chew. Vector potential electromagnetic theory with generalized gauge for inhomogeneous anisotropic media. arXiv:physics$\mathrm{ph} / 1406.4780,2014$.

[13] O. Christensson. An Introduction to Frames and Riesz Bases. Birkhäuser, Boston, 2003.

[14] Tobin A. Driscoll. Schwarz-Christoffel toolbox for Matlab. Available from http://www . math.udel.edu/ driscoll/SC/, 1994-2003.

[15] L. Fishman, A. K. Gautesen, and Z. Sun. An exact, well-posed, one-way reformulation of the Helmholtz equation with application to direct and inverse wave propagation modeling. In New Perspectives on Problems in Classical and Quantum Physics: A Festschrift in Honor of Herbert Überall, pages 75-97. CRC Press, 1998.

[16] Frank Ihlenburg. Finite element analysis of acoustic scattering, volume 132 of Applied Mathematical Sciences. Springer-Verlag, New York, 1998.

[17] D. Ives and J. Liutermoza. Analysis of transonic cascade flow using conformal mapping and relaxation techniques. AIAA Journal, 15(5):647$652,1977$.

[18] J.-M. Jin. Theory and computation of electromagnetic fields. WileyIEEE Press, Hoboken, NJ, USA, 2010.

[19] D. S. Jones. Acoustic and electromagnetic waves. Oxford University press, Oxford, 1986.

[20] D. M. Kerns. Basis of the application of network equations to waveguide problems. J. Res. natn. Bur. Stand., 42:515-540, 1949.

[21] Ren-Cang Li and William Kahan. A family of anadromic numerical methods for matrix Riccati differential equations. Math. Comp., 81(277):233-265, 2012.

[22] J. T. Londegan, J. P. Carini, and D. P. Murdock. Binding and scattering in two-dimensional systems - Applications to quantum wires, waveguides and photonic crystals. Lecture notes in physics. Springer, Berlin, Germany, 1999.

[23] Y. Y. Lu and J. R. Mclaughlin. Propagation in helmholtz waveguides using dtn and ntd maps. Report, City University of Hong Kong, 2000.

[24] Ya Yan Lu. Exact one-way methods for acoustic waveguides. Math. Comput. Simulation, 50(5-6):377-391, 1999. 
[25] A. Maurel, J.-F. Mercier, and S. Félix. Propagation in waveguides with varying cross-section and curvature: A new light on the role of supplementary modes in multimodal methods. Proc. R. Soc. A, 470:20130743, 2014.

[26] R. Mittra and S. W. Lee. Analytical techniques in the theory of guided waves. McMillan, New York, NY, USA, 1971.

[27] N. I. Muskhelishvili. Some basic problems of the mathematical theory of elasticity : Fundamental equations, plane theory of elasticity, torsion and bending. Noordhoff, Leyden, 1962,1975.

[28] A. Nachbin and V. da Silva Simões. Solitary waves in open channels with abrupt turns and branching points. Journal of Nonlinear mathematical Physics, 19:116-136, 2012.

[29] B. Nilsson and O. Brander. The propagation of sound in cylindrical ducts with mean flow and bulk reacting lining - I. Modes in an infinite duct. J. Inst. Maths. Applics, 26:269-298, 1980.

[30] B. Nilsson and O. Brander. The propagation of sound in cylindrical ducts with mean flow and bulk reacting lining - IV. Several interacting discontinuities. IMA J. Appl. Math, 27:263-289, 1981.

[31] Börje Nilsson. Acoustic transmission in curved ducts with various crosssections. Proc. R. Soc. Lond. A, 458:1555-1574, 2002.

[32] J. B. Pendry, D. Schurig, and D. R. Smith. Controlling electromagnetic fields. Science, 312:1780-1782, 2006.

[33] J. Schwartz. Perturbations of spectral operators, and applications, I. bounded perturbations (Lemma 14). Pacific J. of Math., 4:415-458, 1954 .

[34] O. C. Zienkiewicz, R. L. Taylor, and J. Z. Zhu. The Finite Element Method: Its Basis and FundamentalsAnalytical techniques in the theory of guided waves. Butterworth-Heinemann, Oxford, UK, 2013. 


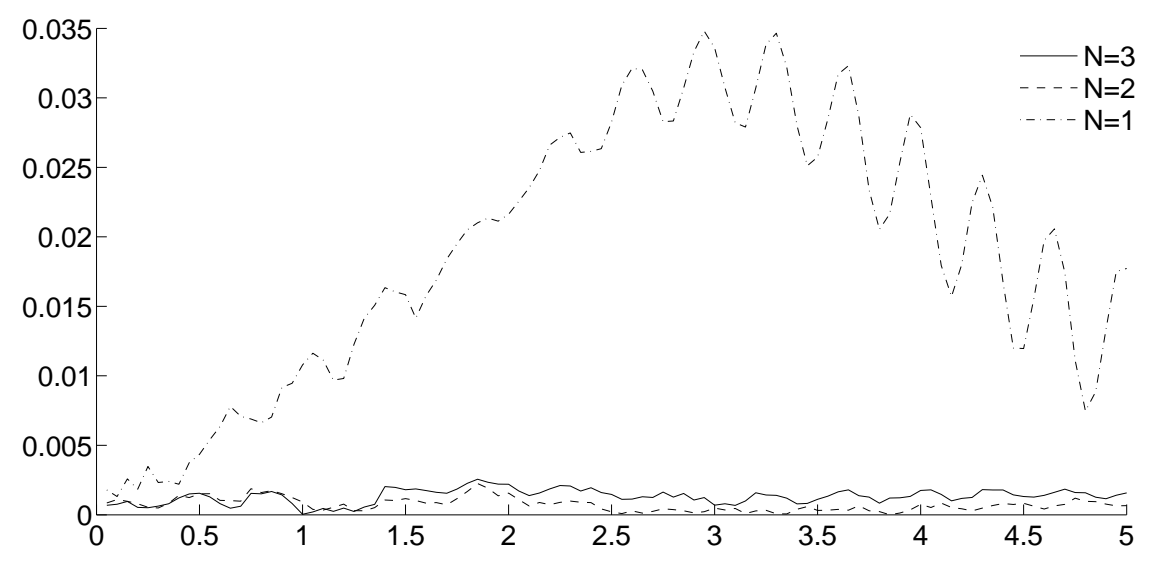

Figure 9: ||$\Phi_{\text {Four }} / \Phi_{\text {FEM }}|-1|$, where $\Phi_{\text {FEM }}$ and $\Phi_{\text {Four }}$ are $\Phi_{0}$ at the end of $\Omega_{3}$ calculated for $0<k \leq 5$ with FE software and Fourier methods respectively. The calculations of $\Phi_{\text {Four }}$ are done with $N=3,2$ and 1, i.e., with $3 \times 3,2 \times 2$ and $1 \times 1$ matrices.

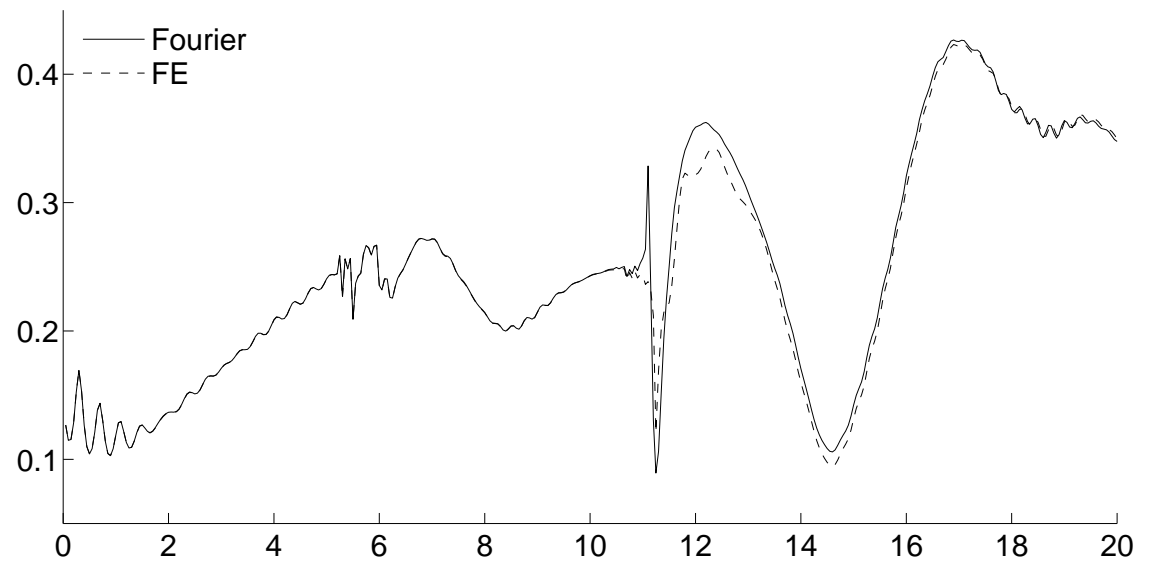

Figure 10: The total field $|\varphi|$ in the point $(7.0671,-1.5855)$ (the left point at the end of the waveguide) for $0<k<20$ calculated with Fourier methods using $N=10$ and with FE software. 


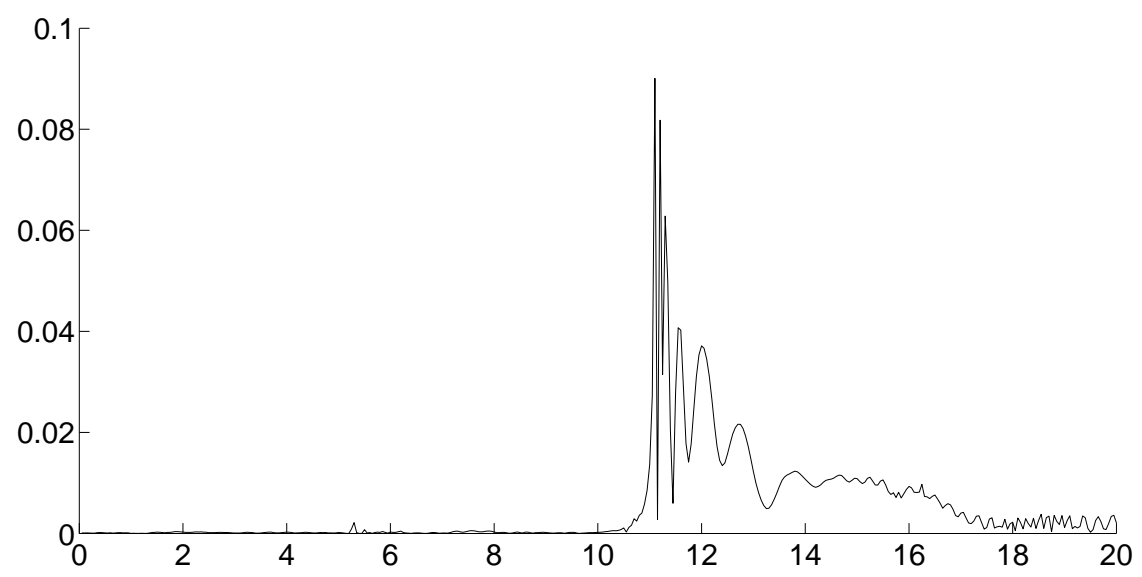

Figure 11: The absolute difference ||$\varphi_{\text {Four }}|-| \varphi_{\mathrm{FE}}||$ for $0<k<20$ in the point $(7.0671,-1.5855)$, where $\varphi_{\text {Four }}$ and $\varphi_{\mathrm{FE}}$ are $\varphi$ calculated with Fourier methods and FE software respectively.

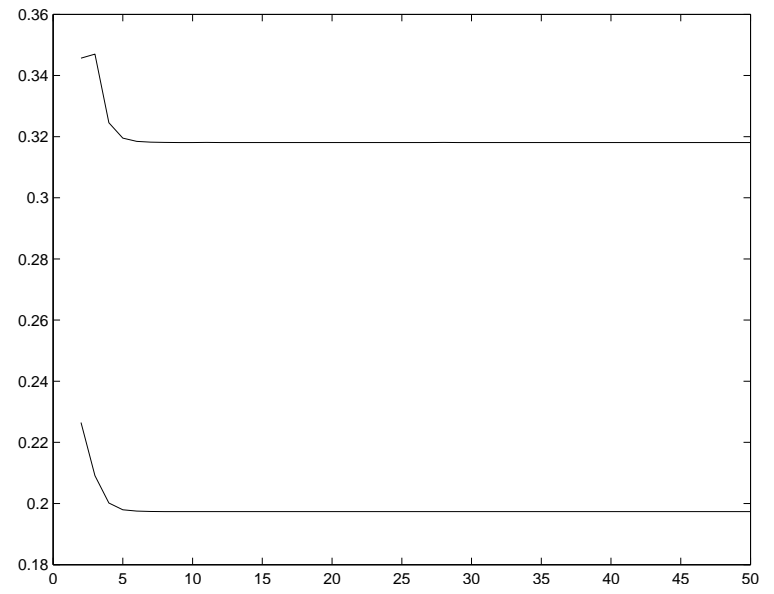

Figure 12: $\Phi_{0}$ and $\Phi_{1}$ at the end of the waveguide calculated for $k=15$ using matrices of size $N \times N$ with $N=2 \ldots 50$. 